\title{
Magnetic properties of nano-scale hematite, -Fe203, studied by time-of-flight inelastic neutron spectroscopy
}

Hill, Adrian H.; Jacobsen, Henrik Skåret; Stewart, J. Ross; Jiao, Feng; Jensen, Niels P.; Holm, Sonja L.; Mutka, Hannu; Seydel, Tilo; Harrison, Andrew; Lefmann, Kim

Published in:

Journal of Chemical Physics

Link to article, DOI:

$10.1063 / 1.4862235$

Publication date:

2014

Document Version

Publisher's PDF, also known as Version of record

Link back to DTU Orbit

Citation $(A P A)$ :

Hill, A. H., Jacobsen, H. S., Stewart, J. R., Jiao, F., Jensen, N. P., Holm, S. L., Mutka, H., Seydel, T., Harrison, A., \& Lefmann, K. (2014). Magnetic properties of nano-scale hematite, - $\mathrm{Fe} \mathrm{O}$, studied by time-of-flight inelastic neutron spectroscopy. Journal of Chemical Physics, 140(4), [044709]. https://aoi.org/10.1063/1.4862235

\section{General rights}

Copyright and moral rights for the publications made accessible in the public portal are retained by the authors and/or other copyright owners and it is a condition of accessing publications that users recognise and abide by the legal requirements associated with these rights.

- Users may download and print one copy of any publication from the public portal for the purpose of private study or research.

- You may not further distribute the material or use it for any profit-making activity or commercial gain

- You may freely distribute the URL identifying the publication in the public portal 


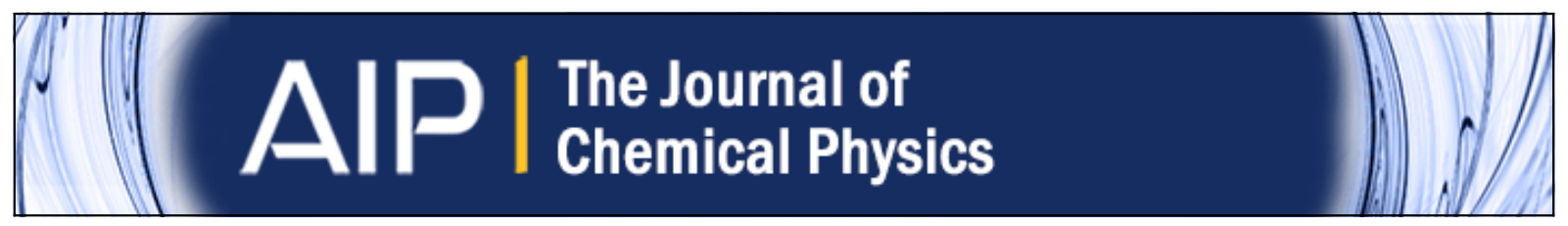

Magnetic properties of nano-scale hematite, -Fe2O3, studied by time-of-flight inelastic neutron spectroscopy

Adrian H. Hill, Henrik Jacobsen, J. Ross Stewart, Feng Jiao, Niels P. Jensen, Sonja L. Holm, Hannu Mutka, Tilo Seydel, Andrew Harrison, and Kim Lefmann

Citation: The Journal of Chemical Physics 140, 044709 (2014); doi: 10.1063/1.4862235

View online: http://dx.doi.org/10.1063/1.4862235

View Table of Contents: http://scitation.aip.org/content/aip/journal/jcp/140/4?ver=pdfcov

Published by the AIP Publishing

\section{AlP Re-register for Table of Content Alerts}




\title{
Magnetic properties of nano-scale hematite, $\alpha-\mathrm{Fe}_{2} \mathrm{O}_{3}$, studied by time-of-flight inelastic neutron spectroscopy
}

\author{
Adrian H. Hill, ${ }^{1, a)}$ Henrik Jacobsen, ${ }^{2, b)}$ J. Ross Stewart, ${ }^{3}$ Feng Jiao, ${ }^{4, c)}$ Niels P. Jensen, ${ }^{5}$ \\ Sonja L. Holm, ${ }^{2}$ Hannu Mutka, ${ }^{6}$ Tilo Seydel, ${ }^{6}$ Andrew Harrison, ${ }^{6,7}$ and Kim Lefmann ${ }^{2}$ \\ ${ }^{1}$ The European Synchrotron Radiation Facility, 6 rue Jules Horowitz, BP220, 38043 Grenoble Cedex 9, France \\ ${ }^{2}$ Nanoscience Centre, Niels Bohr Institute, University of Copenhagen, Universitetsparken 5, 2100 \\ Copenhagen, Denmark \\ ${ }^{3}$ Science and Technology Facilities Council, Rutherford Appleton Laboratory, Harwell Science and Innovation \\ Campus, Didcot OX11 OQX, United Kingdom \\ ${ }^{4}$ Department of Chemical Engineering, University of Delaware, Center for Catalytic Science \& Technology, \\ 150 Academy Street, Newark, Delaware 19716-3110, USA \\ ${ }^{5}$ Department of Energy Conversion and Storage, Technical University of Denmark, Frederiksborgvej 399, \\ DK-4000 Roskilde, Denmark \\ ${ }^{6}$ The Institute Laue-Langevin, 6 rue Jules Horowitz, BP156, 38042 Grenoble Cedex 9, France \\ ${ }^{7}$ EaStCHEM, School of Chemistry and Centre for Science at Extreme Conditions, The King's Buildings, The \\ University of Edinburgh, Mayfield Road, Edinburgh EH9 3JZ, United Kingdom
}

(Received 27 November 2013; accepted 2 January 2014; published online 29 January 2014)

\begin{abstract}
Samples of nanoscale hematite, $\alpha-\mathrm{Fe}_{2} \mathrm{O}_{3}$, with different surface geometries and properties have been studied with inelastic time-of-flight neutron scattering. The $15 \mathrm{~nm}$ diameter nanoparticles previously shown to have two collective magnetic excitation modes in separate triple-axis neutron scattering studies have been studied in further detail using the advantage of a large detector area, high resolution, and large energy transfer range of the IN5 TOF spectrometer. A mesoporous hematite sample has also been studied, showing similarities to that of the nanoparticle sample and bulk $\alpha-\mathrm{Fe}_{2} \mathrm{O}_{3}$. Analysis of these modes provides temperature dependence of the magnetic anisotropy coefficient along the $c$-axis, $\kappa_{1}$. This is shown to remain negative throughout the temperature range studied in both samples, providing an explanation for the previously observed suppression of the Morin transition in the mesoporous material. The values of this anisotropy coefficient are found to lie between those of bulk and nano-particulate samples, showing the hybrid nature of the mesoporous 3-dimensional structure. () 2014 AIP Publishing LLC. [http://dx.doi.org/10.1063/1.4862235]
\end{abstract}

\section{INTRODUCTION}

The chemical and physical properties of materials on a nanoscale are a key area of scientific interest, covering disciplines from theoretical physics through to clean energy sources. The ability to tune desirable properties allows fundamental studies of the underlying physics of the material. Magnetic dynamics provided by magnetic interactions in materials is an area of interest to theoreticians and material scientists alike. Tuning these dynamics can provide novel magnetic materials. One way of altering the material's properties is by reducing the size of individual particles or structures (e.g., thin-films, nanoparticles, nano-structures). Once in the nano-scale region it is widely known that magnetic properties can differ greatly from the material's "bulk" counterpart. ${ }^{1}$

One such material, nanoscale hematite $\left(\alpha-\mathrm{Fe}_{2} \mathrm{O}_{3}\right)$, has shown potential in a variety of fields, in particular its photoelectrical properties. ${ }^{2-4}$ Understanding the underlying dynamics and magnetism may lead to an improved understanding of the material itself, enabling chemists to direct their efforts

\footnotetext{
a) Present address: Johnson Matthey Technology Centre, Savannah, Georgia 31408, USA.

b) hjacobse@fys.ku.dk

c) Previously at: Lawrence Berkeley National Laboratory, California 94720, USA.
}

at materials with specific features. Nano-particulate hematite has been widely studied with a variety of magnetic probes. In particular, inelastic neutron scattering has been used to study high-frequency collective magnetic excitations, enabling calculations of magnetic anisotropy constants in the system, and providing a reason for the suppression of the first order magnetic re-orientation Morin transition (expanded upon below). ${ }^{5-8}$

Another interesting topic in dynamics of nanoparticle magnetism is superparamagnetism (SPM), where the magnetization direction spontaneously reverses direction, assisted by temperature in an Arrhenius fashion. This effect is present also for antiferromagnetic hematite, as was shown directly using Mössbauer spectroscopy ${ }^{9}$ and by quasi-elastic neutron scattering. ${ }^{5,6}$

The relatively recent synthesis of mesoporous (pore sizes between $2 \mathrm{~nm}$ and $50 \mathrm{~nm}$ ) metal oxides ${ }^{10,11}$ with long range crystallographic order provides an entirely new class of material with equally interesting magnetic properties. Because mesoporous materials combine high surface area, nano-sized walls, and three-dimensional coherent structure at a micron meter scale, they may exhibit unique properties, which cannot be observed in nano or bulk counterparts. ${ }^{12-14}$ In this paper we present the first inelastic neutron scattering measurements and analysis from such a material, mesoporous $\alpha-\mathrm{Fe}_{2} \mathrm{O}_{3}$. 
We compare them directly to new measurements made on a sample of hematite nanoparticles (diameter $\approx 15 \mathrm{~nm}$ ), and a bulk reference sample.

\section{A. Properties of "bulk" $\alpha-\mathrm{Fe}_{2} \mathrm{O}_{3}$}

Hematite adopts the corundum structure $(R \overline{3} c$ crystallographic space group) with puckered layers of $\mathrm{Fe}^{3+}$ ions $\left(S=\frac{5}{2}\right)$. It has a Néel temperature at $\approx 955 \mathrm{~K}$, with two ferromagnetic sublattices $\mathbf{s}_{i}$ and $\mathbf{s}_{j}$ coupled antiferromagnetically to each other. The moments align perpendicular to the rhombohedral axis (hexagonal $c$ axis, denoted $x$ ) and lying inside the hexagonal $a b$ basal plane, with a slight canting of the magnetic sublattices towards each other (caused by the Dzyaloshinsky-Moriya interaction, D) leading to weak ferromagnetism (WF-phase). Upon cooling down through $\approx 263 \mathrm{~K}$, a first order magnetic transition occurs, reorientating the magnetic moments to lie almost parallel to the rhombohedral axis. This is known as the Morin transition $\left(T_{\mathrm{M}}\right)$. In the low temperature phase the magnetic moments lie anti-parallel to each other giving a pure antiferromagnetic material (AFM-phase). This spin re-orientation is down to a change in sign of the overall anisotropy of the system made up of $\kappa_{1}$ and $\kappa_{2}$ (Eq. (1)), ${ }^{15-17}$ where $\kappa_{1}$ is related to the anisotropy constant of the (111) direction (the (001) hexagonal direction) and $\kappa_{2}$ is related to the uniaxial anisotropy constant in the basal plane and is positive:

$$
\begin{aligned}
\mathcal{H}= & \sum_{i j}\left(J_{i j} \mathbf{s}_{i} \cdot \mathbf{s}_{j}\right)-\mathbf{D} \cdot \sum_{i j}\left(\mathbf{s}_{i} \times \mathbf{s}_{j}\right) \\
& -\left(\sum_{i} \kappa_{1 i}\left(s_{i}^{x}\right)^{2}+\sum_{i} \kappa_{2 i}\left(s_{i}^{z}\right)^{2}\right) .
\end{aligned}
$$

In the WF-phase, $\kappa_{1}$ is negative, and thus the energy is minimized when the spins lie in the $y z$-plane. Cooling to the AFM-phase, $\kappa_{1}$ changes from negative to positive, and the spins flip to point along the $x$ axis. The change of sign of $\kappa_{1}$ is what causes the Morin transition. The values of $\kappa_{1}$ and $\kappa_{2}$ are related to nanoparticle anisotropy constants $K_{1}$ and $K_{2}$ by $K_{i} V=\kappa_{i} N s^{2}{ }^{7}$ The magnitude of $\kappa_{1}$ is considered several times larger than the $\kappa_{2}$ term, ${ }^{6}$ ignoring the small $T$-interval where $\kappa_{1}$ changes sign.

\section{EXPERIMENTAL}

\section{A. Sample details}

The synthesis and characterization of mesoporous hematite using a mesoporous silica $\left(\mathrm{KIT}-6^{18}\right)$ as a template for decomposition of impregnated $\mathrm{Fe}\left(\mathrm{NO}_{3}\right)_{3} \cdot 9 \mathrm{H}_{2} \mathrm{O}$ been previously described. ${ }^{19}$ The sample has a surface area of $139 \mathrm{~m}^{2} \mathrm{~g}^{-1}$ and a pore diameter centered at $38.5 \AA$. Microscopy images show clear repetition of the mesoporous mosaic, Fig. 1. Crystallographic and magnetic studies (superconducting quantum interference device (SQUID) magnetometry, neutron, and x-ray powder diffraction) ${ }^{19,20,29}$ show that the material adopts the same rhombohedral space group and magnetic structure as the weakly ferromagnetic "bulk" hematite $(263 \mathrm{~K}<T<955 \mathrm{~K})$, and confirm that the sample is of high quality. However, upon cooling down to at least $2 \mathrm{~K}$,
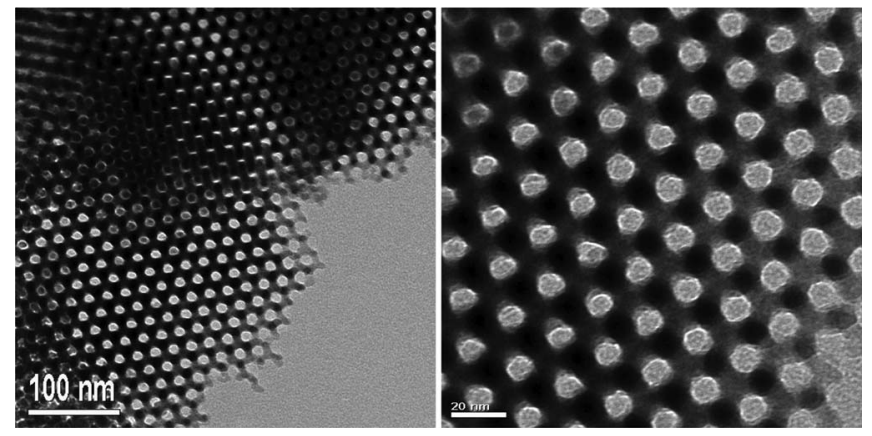

FIG. 1. TEM images of the mesoporous $\alpha-\mathrm{Fe}_{2} \mathrm{O}_{3}$ material. Reprinted with permission from A. H. Hill, F. Jiao, P. G. Bruce, A. Harrison, W. Kockelmann, and C. Ritter, Chem. Mater. 20, 4891 (2008). Copyright 2008 American Chemical Society.

the material does not undergo the Morin transition. This is shown by the continued high intensity of the purely magnetic (003) Bragg peak (hexagonal setting of the $R \overline{3} c$ space group), whose intensity is proportional to the amount of magnetic moment present in the basal $a b$ plane. ${ }^{20}$

The nanoparticulate sample was the same sample used for previous inelastic neutron scattering ${ }^{5}$ and Mössbauer spectroscopy ${ }^{21}$ measurements, with a characteristic size of approximately $15-16 \mathrm{~nm}$. The bulk hematite sample was obtained from Sigma-Aldrich (99.999\%).

\section{B. Time-of-flight (TOF) inelastic neutron scattering and backscattering}

Magnetic dynamics in all three $\alpha-\mathrm{Fe}_{2} \mathrm{O}_{3}$ sample were studied using the cold neutron TOF spectrometer IN5 at the Institut Laue-Langevin (ILL), France. ${ }^{22}$ The sample environment used was a standard orange cryostat, with the sample held in a cylindrical aluminium can. Incident neutron energies of $E_{i}=3.55 \mathrm{meV}\left(\lambda_{i}=4.8 \AA\right)$ and $E_{i}=1.94 \mathrm{meV}\left(\lambda_{i}\right.$ $=6.5 \AA$ ) were selected to provide both large energy transfer and high energy resolution, respectively. The FWHM of the Gaussian resolution functions were determined (from low temperature data) to be $\sim 0.13 \mathrm{meV}$ and $\sim 0.04 \mathrm{meV}$ for $\lambda_{i}$ $=4.8 \AA$ and $\lambda_{i}=6.5 \AA$, respectively.

The momentum transfer $(Q)$ range studied covers the first three Bragg reflections, (003), (101), and (102)), which occur at $1.37 \AA^{-1}, 1.51 \AA^{-1}$, and $1.70 \AA^{-1}$, respectively. Data were taken at various nominal temperatures between $1.5 \mathrm{~K}$ and $300 \mathrm{~K}$.

The quasi-elastic features of the mesoporous and nanoparticulate samples were studied using the backscattering instrument IN10 at the ILL, ${ }^{23}$ with incident energy $E_{i}$ $=2.08 \mathrm{meV}\left(\lambda_{i}=6.27 \AA\right)$. The instrument uses Si (111) analyzers and has 7 detectors, with an energy range of $\pm 12 \mu \mathrm{eV}$. The energy resolution was found from low temperature measurements of the sample to be approximately Gaussian with a FWHM of $1.0 \mu \mathrm{eV}$.

The quasi-elastic features of the nanoparticulate sample were further studied using the newly commissioned backscattering instrument IN16b at the ILL, ${ }^{24,25}$ with incident energy $E_{i}=2.08 \mathrm{meV}\left(\lambda_{i}=6.27 \AA\right)$. The instrument uses Si (111) 

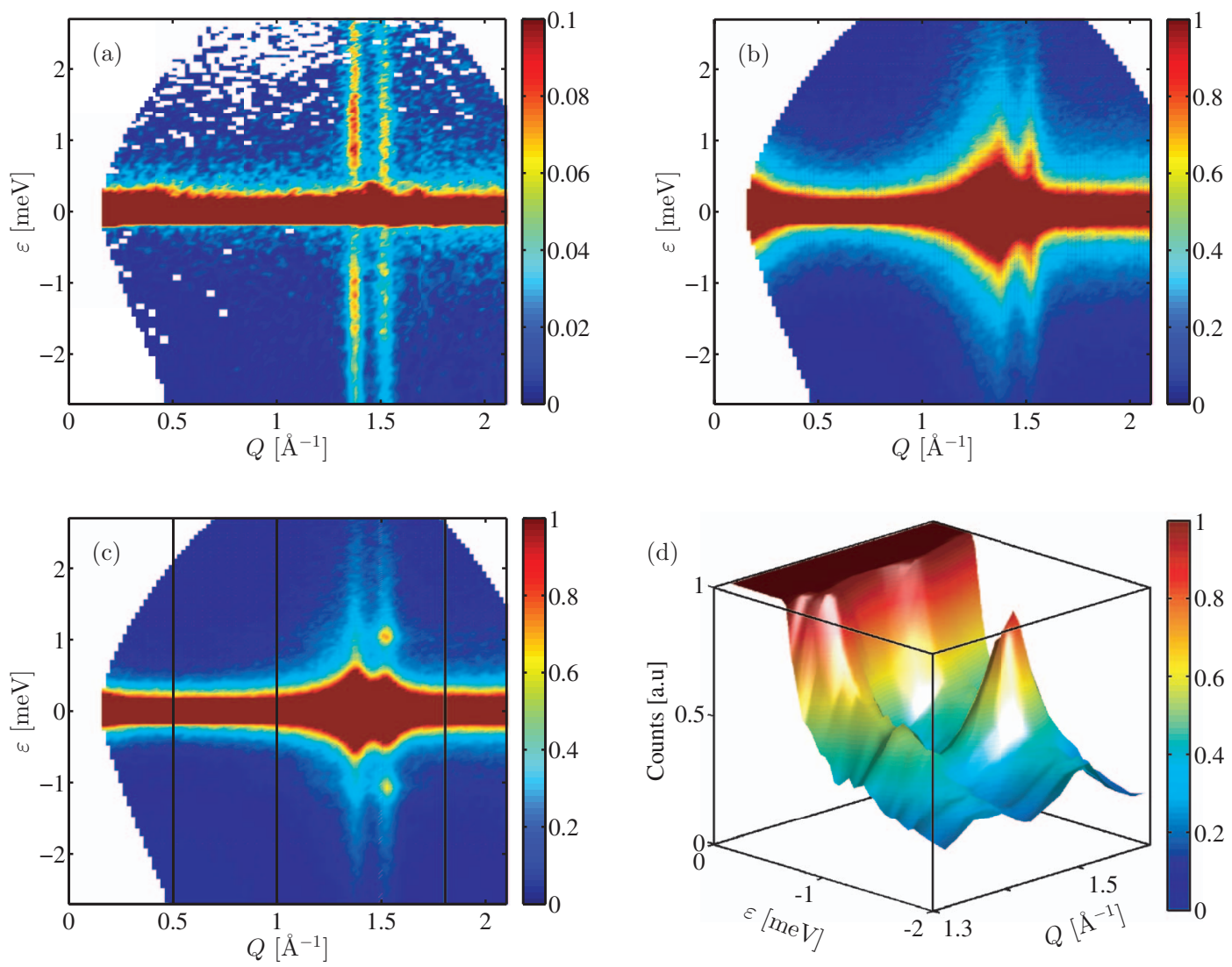

FIG. 2. Spectra of (a) bulk, (b) mesoporous, and (c) nanoparticulate $\alpha-\mathrm{Fe}_{2} \mathrm{O}_{3}$ taken at $150 \mathrm{~K}$ with $\lambda_{i}=4.8 \AA$. Quasi-elastic magnetic peaks and the associated spin-waves at $Q=1.37 \AA^{-1}$ and $Q=1.51 \AA^{-1}$ are clearly visible. These are continuous in the bulk spectrum and quantized in the nanoparticulate spectrum, as seen near $\varepsilon \approx 1.1 \mathrm{meV}$ at $Q=1.51 \AA^{-1}$. The mesoporous spectrum shows behavior between these two states. In (c) the lines indicate the limits within which the incoherent background has been found as described in the text. Panel (d) is a 3D visualization of a part of the data for nanoparticulate $\alpha-\mathrm{Fe}_{2} \mathrm{O}_{3}$ at $300 \mathrm{~K}$.

analyzers and has 16 detectors, with an energy range of $\pm 30 \mu \mathrm{eV}$. The energy resolution was found from low temperature measurements of the sample to be mostly Gaussian with a FWHM of $0.8 \mu \mathrm{eV}$, but with small Lorentzian tails.

The neutron data were converted from time-of-flight to energy using standard treatments (using the Large Array Manipulation Program, LAMP $^{26}$ ) and corrected for the empty aluminum can signal, solid angle and detector efficiency using standard techniques. Example IN5 spectra of the bulk, mesoporous, and nanoparticulate $\alpha-\mathrm{Fe}_{2} \mathrm{O}_{3}$ taken at $150 \mathrm{~K}$ with $\lambda_{i}$ $=4.8 \AA$ are shown in Fig. 2. In bulk hematite, the steep, continuous spin wave dispersion is clearly visible. In contrast to this, the data clearly show the low $\left(\varepsilon_{0}^{+} \approx 0.3 \mathrm{meV}\right)$ and high $\left(\varepsilon_{0}^{-} \approx 1.1 \mathrm{meV}\right)$ frequency inelastic magnetic features of interest at the (003) and (101) Bragg reflections $\left(Q=1.37 \AA^{-1}\right.$ and $Q=1.51 \AA^{-1}$, respectively) in the nanoparticulate and mesoporous spectra.

Examples of the IN10 and IN16b measurements of the nanoparticle sample at various temperatures at $Q=1.37 \AA^{-1}$ are shown in Fig. 3.

\section{RESULTS}

Water adsorbed on the surface of the particles gives rise to large incoherent elastic and quasi-elastic signals. To ac- count for this contribution at the magnetic reflections, all data were initially modeled at non-magnetic $Q$-values, using the following procedure.

At all temperatures, a model (as used in previous studies $^{6-8}$ ) of the incoherent signal (Eq. (2)) was convoluted with the instrument energy resolution to provide the background. The incoherent signal consisted of a sum of an elastic background of area $A_{\mathrm{el}}$; a detailed balance factor, $D(\varepsilon)$ $=\varepsilon(n(\varepsilon)+1)$ corrected quasi-elastic Lorentzian term, area
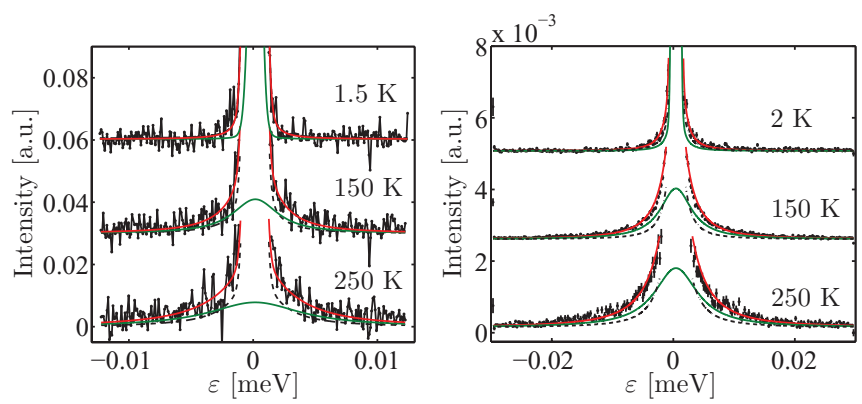

FIG. 3. Example of measurements of the nanoparticle sample at $Q=1.37$ $\AA^{-1}$. (Left) Data from IN10 at $1.5 \mathrm{~K}, 150 \mathrm{~K}$, and $250 \mathrm{~K}$. (Right) Data from IN16b at $2 \mathrm{~K}, 150 \mathrm{~K}$, and $250 \mathrm{~K}$. The dashed (black) line shows the background $I_{\text {incoh }}$ from water, the full (green) line shows the magnetic signal, and the full (red) line shows the fit to Eq. (3). 
$A_{\text {qel }}$ and HWHM $\zeta$; and a constant background, $C$ :

$$
I_{\text {incoh }}=A_{\mathrm{el}} \delta(\varepsilon)+D(\varepsilon)\left(\frac{A_{\text {qel }}}{\pi} \frac{\zeta}{\zeta^{2}+\varepsilon^{2}}\right)+C .
$$

For the IN5 data Eq. (2) was fitted to $Q$ cuts of $\delta(Q)$ $=0.02 \AA^{-1}$, with an energy bin of $0.03 \mathrm{meV}$ and $0.01 \mathrm{meV}$ for incident neutron energies of $E_{i}=3.55 \mathrm{meV}$ and $E_{i}$ $=1.94 \mathrm{meV}$, respectively. Data within $\sim 0.3 \AA$ of $Q$ $=1.37 \AA^{-1}$ and $Q=1.51 \AA^{-1}$ were ignored due to the presence of magnetic scattering, and data at $Q<0.5 \AA^{-1}$ were ignored due to the presence of small angle scattering. The limits are shown with straight lines in Fig. 2(c).

The extracted values of the parameters in Eq. (2) were used to determine $I_{\text {incoh }}$ at $Q=1.37 \AA^{-1}$ and $Q=1.51 \AA^{-1}$. Second order polynomials were used to interpolate the Lorentzian and background $C$ term. The elastic background was interpolated from fitting $A_{\mathrm{el}}$ to a sum of Voigt profiles for the Bragg peaks and a constant background, all multiplied by the Debye-Waller factor.

For the IN10 and IN16b data, a modification of this procedure was used. Due to the smaller amount of detectors, first order polynomials were used to interpolate the Lorentzian and background $C$ term. The elastic background could not be determined with sufficient accuracy, and was thus kept as a free parameter in the following.

The full magnetic signal consists of a superparamagnetic Lorentzian, area $A_{\mathrm{SPM}}$; and the two excitation modes (modeled with two damped harmonic oscillators, DHOs), area $A^{ \pm}$, width $\gamma^{ \pm}$, and position $\varepsilon_{0}^{ \pm}$, providing a model for the total scattering present, Eq. (3), with the incoherent scattering fixed from Eq. (2): ${ }^{6-8}$

$$
\begin{aligned}
I_{\text {model }}= & I_{\text {incoh }} \\
& +D(\varepsilon)\left\{\frac{A_{\mathrm{SPM}}}{\pi} \frac{\Gamma}{\Gamma^{2}+\epsilon^{2}}+\sum_{ \pm} \frac{A^{ \pm}}{\pi} \frac{2 \gamma^{ \pm} \varepsilon_{0}^{ \pm 2}}{\left(\varepsilon^{2}-\varepsilon_{0}^{ \pm 2}\right)^{2}+4 \gamma^{ \pm 2} \varepsilon^{2}}\right\} .
\end{aligned}
$$

\section{A. Nanoparticulate $\alpha-\mathrm{Fe}_{2} \mathrm{O}_{3}$}

As the $15 \mathrm{~nm}$ sample has been well studied previously, although on different instruments, we shall deal with this system first to allow later comparisons to the mesoporous material.

At IN10, data were collected at $1.5 \mathrm{~K}, 50 \mathrm{~K}, 100 \mathrm{~K}$, $150 \mathrm{~K}, 200 \mathrm{~K}, 250 \mathrm{~K}, 300 \mathrm{~K}$, and $350 \mathrm{~K}$. The $\varepsilon_{0}^{ \pm} \mathrm{mag}-$ netic excitations could not be observed within the energy window of IN10, and below around $150 \mathrm{~K}$, almost no broadening of the peaks are seen in either detector, i.e., $A_{\text {qel }} \approx 0$. At $150 \mathrm{~K}$ motion of water sets in and gives rise to a quasielastic broadening in all detectors. Around this temperature, the magnetic signal at $Q=1.37 \AA^{-1}$ also starts to broaden. Above $250 \mathrm{~K}$ the magnetic signal is very weak, and the uncertainty in the elastic background is the same order of magnitude as the magnetic signal. We therefore exclude temperatures above $250 \mathrm{~K}$ in the following. The data were fitted to Eq. (3) without the two inelastic modes.
At IN16b, data were collected at $2 \mathrm{~K}, 150 \mathrm{~K}$, and $250 \mathrm{~K}$ and treated like the IN10 data.

Spectra were taken at IN5 at nominal $1.5 \mathrm{~K}, 50 \mathrm{~K}, 100 \mathrm{~K}$, $150 \mathrm{~K}$, and $300 \mathrm{~K}$ with neutrons with an incident wavelength of $4.8 \AA$ and $1.5 \mathrm{~K}, 50 \mathrm{~K}, 100 \mathrm{~K}, 150 \mathrm{~K}$ with an incident wavelength of $6.5 \AA$.

Inspection of the detailed balance factor in the inelastic data revealed that the sample at nominal $1.5 \mathrm{~K}$ was rather $10 \mathrm{~K}$, which we use in the following. This could be due to slow thermalisation of the sample.

Initial examination of the spectra (an example of which is shown in Fig. 2(c)) clearly shows the high-frequency excitation mode, $\varepsilon_{0}^{-} \approx 1.1 \mathrm{meV}$ at $Q=1.51 \AA^{-1}$ (magnetic (101) Bragg reflection) with signs of the lower frequency excitation, $\varepsilon_{0}^{+}$extending out from the elastic line at both $Q=1.51 \AA^{-1}$ and $Q=1.37 \AA^{-1}$ (the (101) and (003) magnetic Bragg reflections). Looking carefully at the (003) reflection, it is also possible to observe a small amount of the high frequency mode, Fig. 2(d), as also seen by Klausen et $_{\text {al. }}{ }^{7}$

Slices of the spectra were taken across $Q$ at $1.37 \AA^{-1}$ and $1.51 \AA^{-1}$, with a $\Delta Q$ of $0.02 \AA^{-1}$ and subsequently fitted to the model presented in Eq. (3). The superparamagnetic broadening is so small that it cannot be measured with the resolution of IN5, so the $A_{\text {spm }}$ term was replaced with a delta function of area $A_{\mathrm{spm}}$.

Example fits of both cuts are shown in Fig. 4. The low frequency mode is seen as a broad shoulder to the elastic line and the high frequency mode is seen as a peak near $\sim \pm 1.1 \mathrm{meV}$. Poor agreement between the model and observed scattering is obtained at high energy transfers, probably due to higher order excitations not modeled here. As a consequence of this, the high frequency mode was only modeled in the total fit for the $Q=1.51 \AA^{-1}$ data.
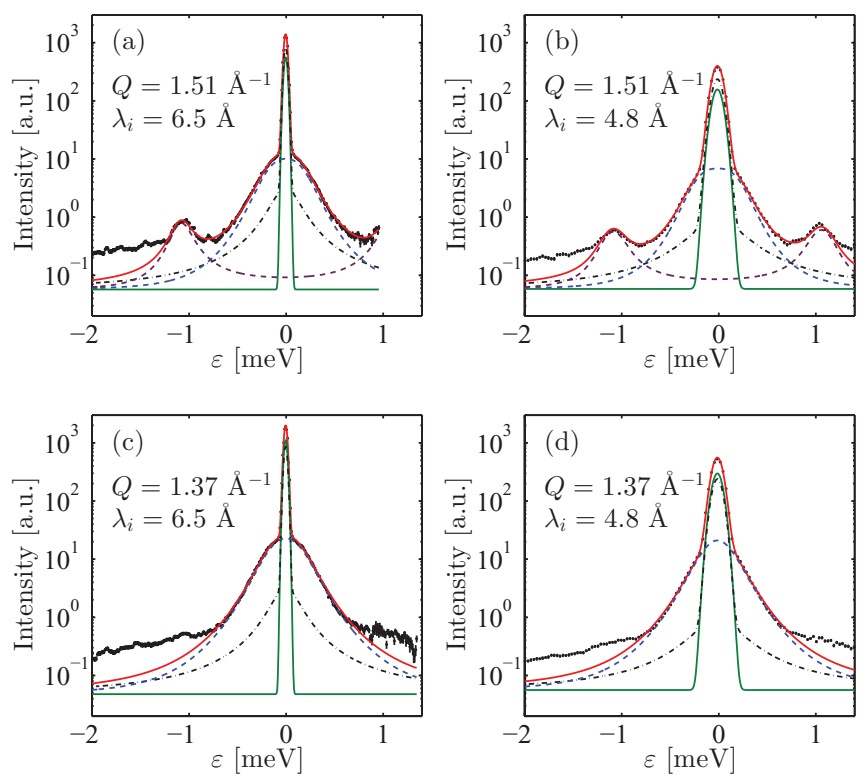

FIG. 4. Fits of cuts in the nanoparticulate data from IN5 at (a) $Q=1.51 \AA^{-1}$, $\lambda_{i}=6.5 \AA$, (b) $Q=1.51 \AA^{-1}, \lambda_{i}=4.8 \AA$, (c) $Q=1.37 \AA^{-1}, \lambda_{i}=6.5 \AA$, and (d) $Q=1.37 \AA^{-1}, \lambda_{i}=4.8 \AA$. The total fit is shown with a broad (red) line, the two DHOs are shown with dashed (blue and magenta) lines, the SPM is shown with a solid (green) line, and $I_{\text {incoh }}$ shown with a dashed-dotted (black) line. $T=150 \mathrm{~K}$. 


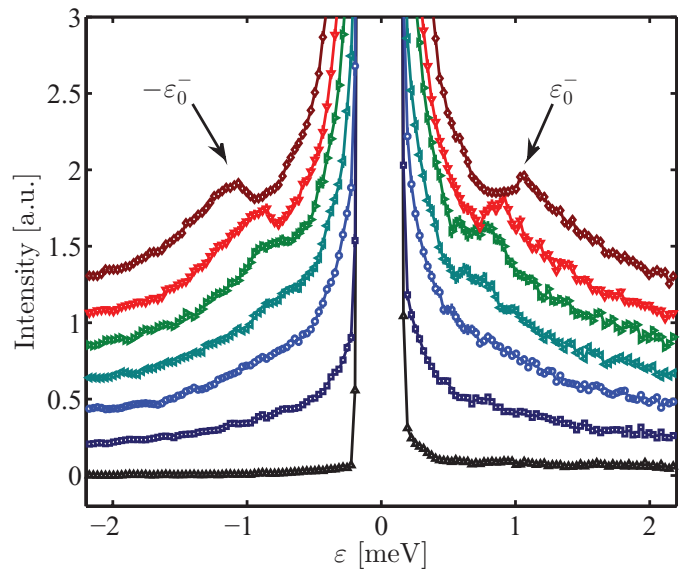

FIG. 5. Temperature dependence of the mesoporous $\alpha-\mathrm{Fe}_{2} \mathrm{O}_{3}$ spectra at $Q=1.51 \AA^{-1}$, with $\lambda_{i}=4.8$, showing the evolution of the high frequency mode at $\approx 1 \mathrm{meV}(300 \mathrm{~K})$. Spectra are arranged from $300 \mathrm{~K}$ (top) in steps of $50 \mathrm{~K}$ to $10 \mathrm{~K}$ (bottom) offset from each other for clarity.

\section{B. Mesoporous $\alpha-\mathrm{Fe}_{2} \mathrm{O}_{3}$}

Backscattering data at IN10 were collected at $1.5 \mathrm{~K}$, $50 \mathrm{~K}, 100 \mathrm{~K}, 150 \mathrm{~K}, 200 \mathrm{~K}, 250 \mathrm{~K}, 300 \mathrm{~K}, 350 \mathrm{~K}$, and $400 \mathrm{~K}$. At $150 \mathrm{~K}$ motion of water sets in and gives rise to a quasielastic broadening in all detectors. The data were fitted to Eq. (3) without the two inelastic modes.

Spectra of the mesoporous sample were taken at IN5 at $1.5 \mathrm{~K}, 50 \mathrm{~K}, 100 \mathrm{~K}, 150 \mathrm{~K}, 200 \mathrm{~K}, 250 \mathrm{~K}$, and $300 \mathrm{~K}$ with neutrons with incident wavelengths of $4.8 \AA$ and $6.5 \AA$. The example spectra in Fig. 2(b) shows significant broadening of the elastic line, and considerable inelastic scattering away at the (003) and (101) Bragg reflections.

Only the $\varepsilon_{0}^{-}$mode is clearly visible at approximately $1 \mathrm{meV}$ at $300 \mathrm{~K}$, Fig. 5. To fit the mesoporous spectra, a similar strategy to that used on the nanoparticle analysis was applied, however despite numerous attempts, no reliable fit could be performed. Therefore, we have estimated the energy of the high frequency mode by eye at $Q=1.51 \AA^{-1}$ (using both the up and down scattering sides with $\lambda_{i}=4.8 \AA$, and the down scattering side with $\lambda_{i}=6.5 \AA$ ). No sign of the high frequency mode was seen at $Q=1.37 \AA^{-1}$.

\section{ANALYSIS}

Two types of magnetic dynamics are investigated here: superparamagnetic spin flips and $Q=0$ spin waves. We first look at the superparamagnetism.

The average time between spin flips, $\tau$ is highly temperature dependent as described by the Néel-Brown law: ${ }^{1,5,6}$

$$
\tau=\tau_{0} e^{K_{2} V / k_{B} T},
$$

where $\tau_{0}$ is slightly temperature dependent, ${ }^{27}$ but usually taken as a constant. $\tau$ is related to the observed Lorentzian broadening by $\Gamma=\hbar / \tau$.

The extracted values of $\Gamma$ from the IN10 and IN16b experiments on the nanoparticles are shown in Fig. 6. No signs of superparamagnetic spin flips are seen until $\sim 150 \mathrm{~K}$. Above $250 \mathrm{~K}$ the extracted widths are unreliable because of the large incoherent background and the fact that the magnetic signal

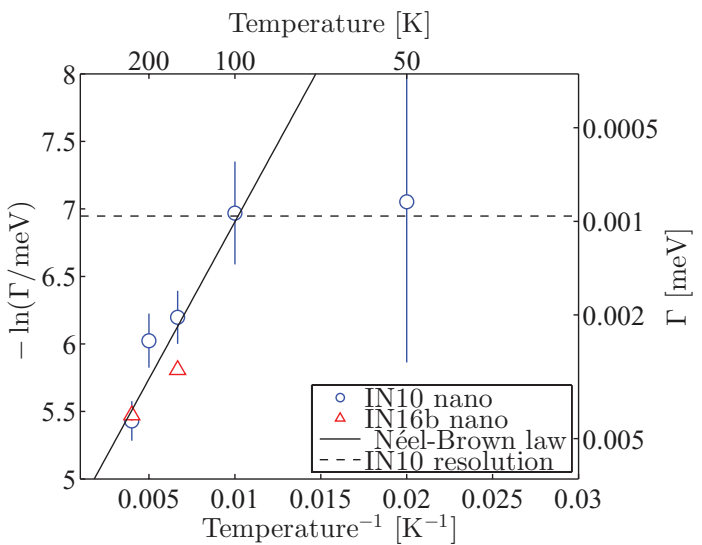

FIG. 6. Width of magnetic peak measured at IN10 and IN16B. Above $250 \mathrm{~K}$ the IN10 data are unreliable because of difficulties in fixing the large incoherent background, and they are thus not shown.

has broadening so much that it is barely visible within the energy window of IN10 $( \pm 12 \mu \mathrm{eV})$.

The data between $100 \mathrm{~K}$ and $250 \mathrm{~K}$ have been fitted to Eq. (4), yielding $K_{2} V / k_{B}=230(70) \mathrm{K}$ and $\tau_{0}=6.4(6)$ $\times 10^{-11} \mathrm{~s}$. The value of $K_{2} V / k_{B}$ is lower than reported previously for these particles. ${ }^{5,6}$

In the mesoporous particles, no broadening of the magnetic signal is seen within the resolution of IN10, confirming the absence of superparamagnetic behavior in this sample, as observed previously with Mössbauer measurements. ${ }^{19}$

To model the excitations, a simplified zero-field spin wave dispersion relationship for hematite has previously been described and shall be used here, Eq. (5): ${ }^{7}$

$$
\pm \varepsilon_{0}^{ \pm} \approx 2 \frac{\left\langle S^{2}\right\rangle}{S} \sqrt{\left(\mathcal{A}+\mathcal{J}_{0} \pm \mathcal{J}_{q}\right)\left(\mathcal{B}+\mathcal{J}_{0} \mp \mathcal{J}_{q}\right)} .
$$

Calculation of the variables $\mathcal{A}$ and $\mathcal{B}$ enables the extraction of the anisotropy coefficients $\kappa_{1}$ and $\kappa_{2}$ using the relationships $\mathcal{A}=\left(-\kappa_{1}+\kappa_{2}\right) s^{\prime}$ and $\mathcal{B}=\kappa_{2} s^{\prime}$ with $s^{\prime}=S-1 / 2$. The extracted positions of the low and high energy excitations $\varepsilon_{0}^{ \pm}$ are used with the mean ordered spin moment, $\left\langle S^{z}\right\rangle$ to determine $\mathcal{A}$ and $\mathcal{B}$, Eq. (6):

$$
\begin{aligned}
& \varepsilon_{0}^{+} \approx 2 \frac{\left\langle S^{z}\right\rangle}{S} \sqrt{2 \mathcal{J}_{0} \mathcal{B}}, \\
& \varepsilon_{0}^{-} \approx 2 \frac{\left\langle S^{z}\right\rangle}{S} \sqrt{2 \mathcal{J}_{0} \mathcal{A}} .
\end{aligned}
$$

The value of $\mathcal{J}_{0}$ can be determined from nearest neighbor out-of-plane exchange constants and the lattice parameter $c$ (hexagonal) in the [001] direction, knowing that as $Q \rightarrow$ $0 \mathcal{J}_{0}=\mathcal{J}_{Q}=-S\left(J_{1}+3 J_{3}+6 J_{4}\right) \cos (c Q)$. The exchange constants have been previously calculated by Samuelsen and Shirane $\left(J_{1}=6 \mathrm{~K}, J_{3}=-29.7 \mathrm{~K}\right.$, and $\left.J_{4}=-23.2 \mathrm{~K}\right){ }^{28}$

With knowledge of the ordered moment $\left\langle S^{z}\right\rangle$, we can thus use the measured values of $\varepsilon_{0}^{ \pm}$to determine the values of $\kappa_{1}$ and $\kappa_{2}$.

There are two ways of obtaining $\left\langle S^{z}\right\rangle$ as function of temperature. It may be calculated using Boltzmann statistics as in, e.g., Refs. 6 and 27. This, however, requires knowledge of the energy barrier $K V=N_{2} s^{2}$, which is unknown in the mesoporous particles. The second method is to utilize the fact that at the magnetic reflections, $\mathbf{Q}=\mathbf{G}$, where $\mathbf{G}$ referes to 


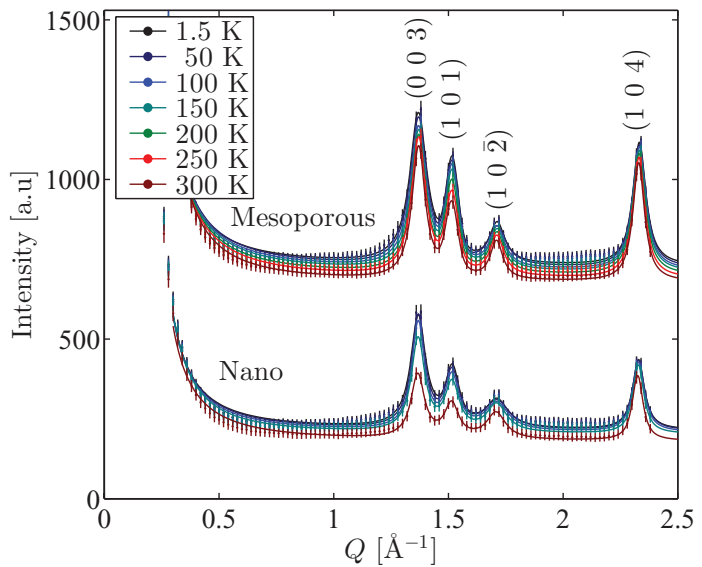

FIG. 7. The measured signal at $\varepsilon=0$ (bin width $=0.02 \mathrm{meV}$ ) as function of $Q$ for the nanoparticles (bottom) and the mesoporous particles (top). The two datasets have been displaced by a constant for clarity; the incoherent background has roughly the same magnitude for the two sets of data.

the (003) and (101) reflections, the elastic scattering is proportional to the square of the ordered moment:

$$
I(\varepsilon=0,|Q|=|\tau|) \propto\left\langle S^{z 2}\right\rangle \approx\left\langle S^{z}\right\rangle^{2} .
$$

The data at $\varepsilon=0$ are shown in Fig. 7. The solid lines through the data are fits to Voigt functions for the peaks plus a constant background multiplied by the Debye-Waller factor. The extracted temperature dependence of $\left\langle S^{z}\right\rangle$ is approximately linear and is shown in Fig. 8 together with the measured values of $\varepsilon_{0}^{ \pm}$for the nanoparticles and $\varepsilon_{0}^{-}$for the mesoporous particles. The temperature dependence of $\varepsilon_{0}^{+}$is accurately described by this model, implying that the corresponding anisotropy, $\kappa_{2}$ is independent of temperature. The same cannot be said of $\varepsilon_{0}^{-}$, whose temperature dependence is caused not only by $\left\langle S^{z}\right\rangle$, but also from a temperature dependence of $\kappa_{1}$ as shown in Ref. 7.

To find the temperature dependence of $\kappa_{1}$ we first extract $\kappa_{2}$ from the measurement of $\varepsilon_{0}^{+}$, using Eq. (6a) and the measured values of $\left\langle S^{z}\right\rangle / S$. We find $\kappa_{2}=1.49$ (3) $\mathrm{mK}$ in accordance with previous work. ${ }^{7}$ Subsequently, Eq. (6b) has been used to find $\kappa_{1}$. In Ref. 7 the approximation $\left\langle S^{z}\right\rangle / S \approx 1$ is

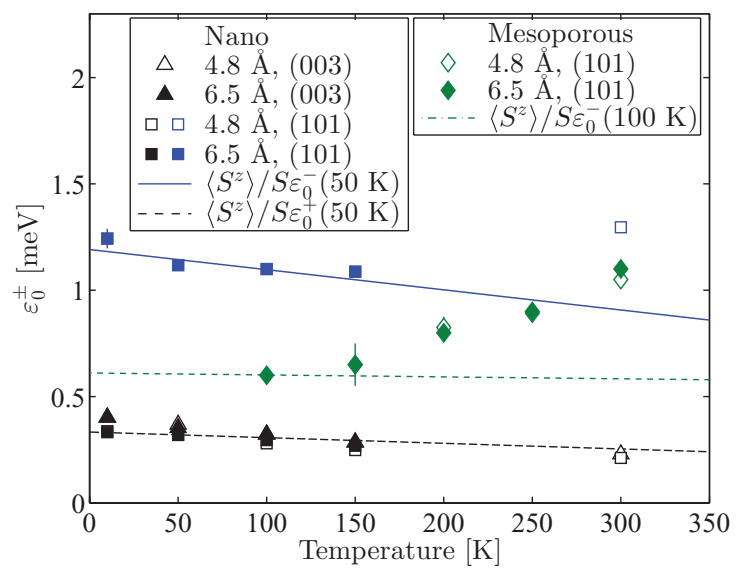

FIG. 8. The measured values of $\varepsilon_{0}^{ \pm}$. The solid lines show the temperature evolution of $\left\langle S^{z}\right\rangle / S$ as determined from the elastic data. The increase of $\varepsilon_{0}^{-}$ with temperature is due to the increase of $\left|\kappa_{1}\right|$.

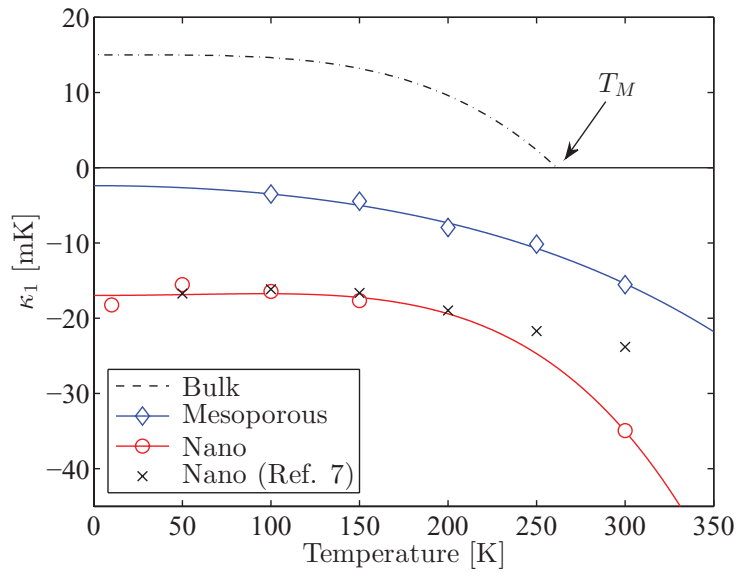

FIG. 9. The values of $\kappa_{1}$ for bulk, nanoparticulate and mesoporous hematite determined using Eqs. (6a) and (6b). The error bars are smaller than the point size. The dashed line shows the value for bulk hematite (taken from Ref. 7), and the crosses are the results for nanoparticles from Ref. 7, which uses the approximation $\left\langle S^{z}\right\rangle / \mathrm{S}=1$, see discussion in text.

made. This is a fair approximation at low temperatures, but about $15 \%$ off at $300 \mathrm{~K}$. Our values of $\left|\kappa_{1}\right|$ are therefore larger than those reported in Ref. 7. We find $\kappa_{1}$ to vary between $\sim-15 \mathrm{mK}$ at $50 \mathrm{~K}$ and $\sim-35 \mathrm{mK}$ at $300 \mathrm{~K}$. The extracted values of $\kappa_{1}$ are shown in Fig. 9 along with the data from Ref. 7. The solid lines are empirical models of the temperature dependence of $\kappa_{1}$ as an even fourth order polynomial in $T$. In Ref. 7 a second order polynomial is used. With the improved estimate of $\left\langle S^{z}\right\rangle / S$ the fourth order polynomial gives a better fit to the data with the same amount of free parameters.

At low temperatures, the relative amplitude of the SPM signal to the excitations is given by ${ }^{6}$

$$
\begin{aligned}
\frac{A_{\mathrm{SPM}}}{A_{\mathrm{SPM}}+A_{2}}= & \frac{I_{0}\left(\alpha_{2} / 2\right)+I_{1}\left(\alpha_{2} / 2\right)}{2 I_{0}\left(\alpha_{2} / 2\right)} \\
& \times \frac{\left.\alpha_{1}^{-1 / 2} \mathrm{e}^{-\alpha_{1}}+1 / 2 \sqrt{\pi}\left(2-\alpha_{1}^{-1}\right) \operatorname{erf}\left(\sqrt{\alpha_{1}}\right)\right)}{\sqrt{\pi} \operatorname{erf}\left(\sqrt{\alpha_{1}}\right)}
\end{aligned}
$$

$$
\approx 1-k_{\mathrm{B}} T\left(\frac{1}{2\left|K_{1} V\right|}+\frac{1}{2\left|K_{2} V\right|}\right)
$$

where $\alpha_{i}=K_{i} V / k_{\mathrm{B}} T, I_{n}(x)$ is the modified Bessel function of order $n$, and $\operatorname{erf}(x)=2 / \sqrt{\pi} \int_{0}^{x} \mathrm{e}^{-u^{2}} \mathrm{~d} u$ is the error function.

Using $K_{1} V=\left|\kappa_{1}\right| s^{2} N$ and the measured value of $\kappa_{1}$ at low temperatures, with $N \approx 10^{5}$, we find $K_{2} V=590(20) \mathrm{K}$, meaning $\kappa_{2}=0.94(3) \mathrm{mK}$. The fit is shown in Fig. 10. This value is smaller than the value obtained from the measured value of $\kappa_{2}=1.49(3) \mathrm{mK}, K_{2} V=\left|\kappa_{2}\right| s^{2} N=936(20) \mathrm{K}$. Using this value produces the dashed green line in Fig. 10. The error bars are the statistical errors from the fits, and the discrepancy between theory and measurements is therefore not because of uncertainties in the data.

As shown in Fig. 2(d) the high frequency mode is also visible at $Q=1.37 \AA^{-1}$. To extract the amplitude the data near the high frequency mode were fitted to a Gaussian on a sloping background, with position and width fixed to the position and width at $Q=1.51 \AA^{-1}$. The resulting relative 


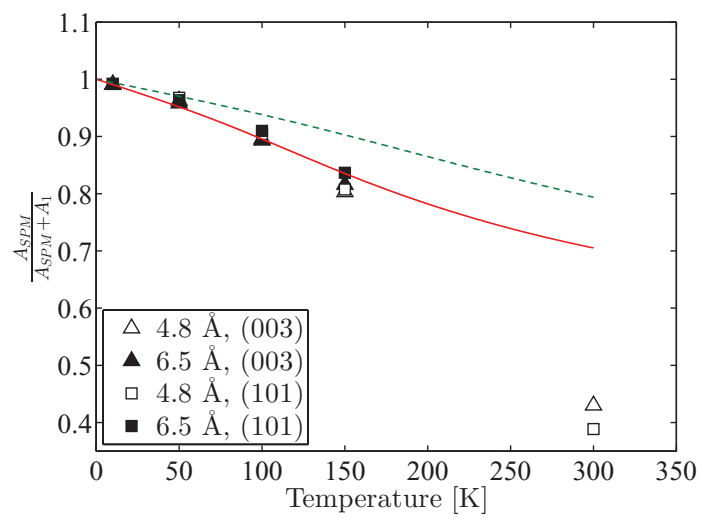

FIG. 10. The relative area of the magnetic quasielastic signal to the total magnetic signal for the nanoparticles. The solid red line is the fit given by Eq. (9), the dashed green line is the expected behavior with the values of $\kappa_{1}$ and $\kappa_{2}$ obtained here.

areas are shown in Fig. 11. The large error bars are due to the very low signal at $Q=1.37 \AA^{-1}$. In Ref. 7 the ratio is found to be $\approx 0.11$, which is fairly consistent with our data. Interestingly, the data from the two different wavelengths do not agree here.

For the mesoporous particles the analysis is not so straightforward. We cannot resolve the low frequency mode, and since the sample does not perform superparamagnetic spin flips we cannot determine $\kappa_{2}$ with our data. Since the value of $\epsilon_{0}^{+}$is roughly the same for nanoparticles and bulk, $5,6,8,15,21$ it is a fair approximation to also use this value for the mesoporous particles. The values of $\varepsilon_{0}^{-}$are shown in Fig. 8 down to $100 \mathrm{~K}$, below which the mode cannot be seen. As for the nanoparticles, the energy increases and does not follow the value of $\left\langle S^{z}\right\rangle / S$ which stays nearly constant over the observed temperature range.

Repeating the above described analysis we find $\kappa_{1}$ to vary between $\sim-3 \mathrm{mK}$ at low temperatures and $\sim-15 \mathrm{mK}$ at $300 \mathrm{~K}$. This is summarized in Fig. 9, where also the bulk value of $\kappa_{1}$ is shown. ${ }^{7,15}$

From Fig. 9, we can clearly see that $\kappa_{1}$ in mesoporous hematite does not change to a positive value down to $2 \mathrm{~K}$.

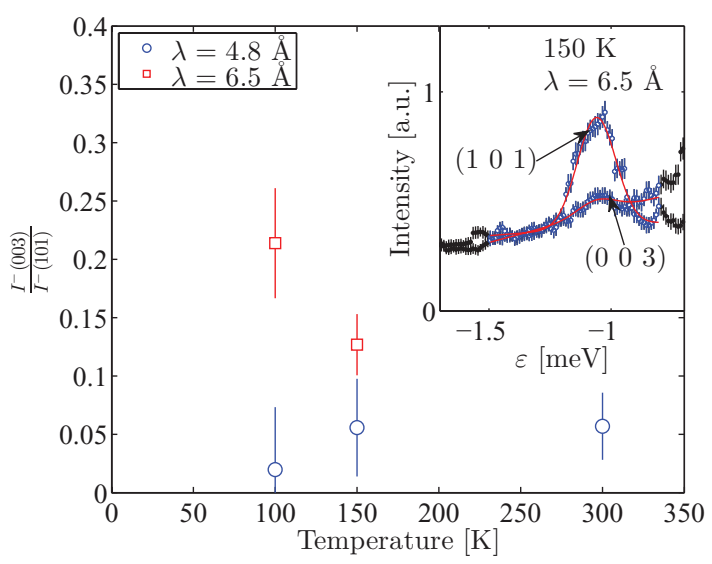

FIG. 11. The relative amplitude of the high frequency mode at (003) and (101). Below $100 \mathrm{~K}$ the signal is not visible above the background at (003). The inset shows an example of the fit.
Rather, it follows a similar trend to the nano-particulate hematite value. At low temperatures, the $\kappa_{1}$ value of the mesoporous hematite sample is much closer to $0 \mathrm{mK}$ than the values reported for nanoparticle hematite. This suggests that it may be possible to observe the Morin transition in a mesoporous material engineered with slightly thicker walls, making a more "bulk" like material.

Previously, the spin waves in nanoparticles have been measured as being completely symmetric with no tails to the high energy side. ${ }^{7}$ However, due to the high intensity of the IN5 spectrometer, we are able to see hints of a tail towards the high energy side. Plots suggest that for energy transfers larger than $1.2 \mathrm{meV}$, the same theory is applicable to all 3 forms of hematite.

\section{CONCLUSION}

Throughout the range of $T$ studied we can see that the values of $\kappa_{1}$ decrease from "bulk" $\gg$ mesoporous $>$ nanoparticle material. The lack of change in sign of $\kappa_{1}$ explains the suppression of the Morin transition observed in earlier studies of the mesoporous hematite sample. The fact that $\kappa_{1}$ lies between values of "bulk" and nanoparticle samples is indicative of the nature of the structure of the material. Nanoparticles have no long range order between particles, whereas the mesoporous material is a 3-dimensional solid over much larger distances, bridging the gap between bulk and nanomaterials, as shown in X-ray pair distribution function (PDF) measurements and modelling. ${ }^{29}$ The present study furthers our knowledge of mesoporous $\alpha-\mathrm{Fe}_{2} \mathrm{O}_{3}$ and other related materials, which too show magnetic properties slightly different from bulk material. ${ }^{30}$

\section{ACKNOWLEDGMENTS}

Neutron scattering experiments in this project were carried out at the ILL. The project was funded by the Danish Research Council for Nature and Universe through DANSCATT.

We thank Sonja Rosenlund Hansen for assistance in the early phases of this project, and Franz Bødker for providing the nanoparticle sample.

${ }^{1}$ L. Néel, C. R. Hebd. Seances Acad. Sci. 252, 4075 (1961).

${ }^{2}$ V. R. Satsangia, S. Kumaria, A. P. Singha, R. Shrivastavb, and S. Dass, Int. J. Hydrogen Energ. 33, 312 (2008).

${ }^{3}$ D. K. Bora, A. Braun, and E. C. Constable, Energy Environ. Sci. 6, 407 (2013).

${ }^{4}$ D. A. Wheeler, G. Wang, Y. Ling, Y. Li, and J. Z. Zhang, Energy Environ. Sci. 5, 6682 (2012).

${ }^{5}$ M. F. Hansen, F. Bødker, S. Mørup, K. Lefmann, K. N. Clausen, and P.-A. Lindgård, Phys. Rev. Lett. 79, 4910 (1997).

${ }^{6}$ S. N. Klausen, K. Lefmann, P.-A. Lindgård, K. N. Clausen, M. F. Hansen, F. Bødker, S. Mørup, and M. Telling, J. Magn. Magn. Mater. 266, 68 (2003).

${ }^{7}$ S. N. Klausen, K. Lefmann, P.-A. Lindgård, L. Theil Kuhn, C. R. H. Bahl, C. Frandsen, S. Mørup, B. Roessli, N. Cavadini, and C. Niedermayer, Phys. Rev. B 70, 214411 (2004).

${ }^{8}$ L. Theil Kuhn, K. Lefmann, C. R. H. Bahl, S. N. Ancona, P.-A. Lindgård, C. Frandsen, D. E. Madsen, and S. Mørup, Phys. Rev. B 74, 184406 (2006).

${ }^{9}$ W. Kündig, H. Bömmel, G. Constabaris, and R. H. Lindquist, Phys. Rev. 142, 327 (1966).

${ }^{10}$ W. B. Yue and W. Z. Zhou, Prog. Nat. Sci. 18, 1329 (2008). 
${ }^{11}$ Y. Ren, Z. Ma and P. G. Bruce, Chem. Soc. Rev. 41, 4909 (2012).

${ }^{12}$ W. Xuan, C. Zhu, Y. Liu, and Y. Cui, Chem. Soc. Rev. 41, 1677 (2012).

${ }^{13}$ K. Ariga, A. Vinu, Y. Yamauchi, Q. Ji, and J. P. Hill, Bull. Chem. Soc. Jpn. 85, 1 (2012).

${ }^{14}$ C. Perego and R. Millini, Chem. Soc. Rev. 42, 3956 (2013).

${ }^{15}$ A. H. Morrish, Canted Antiferromagnetism: Hematite (World Scientific, Singapore, 1994).

${ }^{16}$ C. R. H. Bahl, J. Garde, K. Lefmann, T. B. S. Jensen, P.-A. Lindgård, D. Madsen, and S. Mørup, Eur. Phys. J. B 62, 53 (2008).

${ }^{17}$ H. Chow and F. Keffer, Phys. Rev. B 10, 243 (1974).

${ }^{18}$ F. Kleitz, S. H. Choi, and R. Ryoo, Chem. Commun. 2136 (2003).

${ }^{19}$ F. Jiao, A. Harrison, J. C. Jumas, A. V. Chadwick, W. Kockelmann, and P. G. Bruce, J. Am. Chem. Soc. 128, 5468 (2006).

${ }^{20}$ A. H. Hill, F. Jiao, P. G. Bruce, A. Harrison, W. Kockelmann, and C. Ritter, Chem. Mater. 20, 4891 (2008).
${ }^{21}$ F. Bødker, M. F. Hansen, C. B. Koch, K. Lefmann, and S. Mørup, Phys. Rev. B 61, 6826 (2000).

${ }^{22}$ J. Ollivier, H. Mutka, and L. Didier, Neutron News 21, 22 (2010).

${ }^{23}$ O. G. Randl, H. Franz, T. Gerstendörfer, Q. Petry, G. Vogl, and A. Magerl, Physica B 234-236, 1064 (1997).

${ }^{24}$ B. Frick, H. N. Bordallo, T. Seydel, J.-F. Barthélémy, M. Thomas, D. Bazzoli, and H. Schober, Physica B 385-386, 1101 (2006).

${ }^{25}$ B. Frick, E. Mamontov, L. V. Eijck, and T. Seydel, Z. Phys. Chem. 224, 33 (2010).

${ }^{26}$ D. Richard, M. Ferrand, and G. J. Kearley, J. Neutron Res. 4, 33 (1996).

${ }^{27}$ M. F. Hansen, F. Bødker, S. Mørup, K. Lefmann, K. N. Clausen, and P.-A. Lindgård, J. Magn. Magn. Mater. 221, 10 (2000).

${ }^{28}$ E. J. Samuelsen and G. Shirane, Phys. Status Solidi B 42, 241 (1970).

${ }^{29}$ A. H. Hill and M. Allieta, Phys. Chem. Chem. Phys. 15, 8583 (2013).

${ }^{30}$ A. H. Hill, A. Harrison, C. Dickinson, W. Zhou, and W. Kockelmann, Microporous Mesoporous Mater. 130, 280 (2010). 